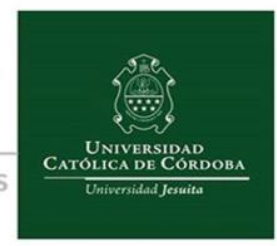

CASO CLINICO Rev. Methodo 2022;7(1):37-41

https://doi.org/10.22529/me.2022.7(1)06

Recibido 29 Jul. 2021| Aceptado 27 Oct. 2021|Publicado 05 Ene. 2022

\title{
Trombocitopenia inmune primaria: reporte de casos no respondedores al tratamiento
}

\section{Primary immune thrombocytopenia: report of cases nonresponders to treatment}

\author{
Florencia Belén Cabrera $^{1}$ (iD), Valentina Bressan ${ }^{1}$, Pablo Lucero $^{1}$, Ignacio Gutiérrez Magaldi ${ }^{1}$, \\ Mario Luciano Salvano ${ }^{2}$, Florencia Gazzoni². \\ 1. Universidad Católica de Córdoba, Facultad de Ciencias de la Salud, Clínica Universitaria Reina Fabiola, Servicio de Medicina Interna \\ 2. Universidad Católica de Córdoba, Facultad de Ciencias de la Salud, Clínica Universitaria Reina Fabiola, Servicio de Hematología \\ Correspondencia: Florencia Belén Cabrera, e-mail: floppycabrera@gmail.com
}

\section{Resumen}

La trombocitopenia inmune primaria (PTI) es una patología rara caracterizada por trombocitopenia aislada de origen autoinmune, con un recuento $<100$ x 109 plaquetas/l no asociado a una patología reconocible, que cursa con un aumento del riesgo de hemorragia. No existe ninguna prueba confirmatoria definitiva para establecer su diagnóstico, que se realiza fundamentalmente por exclusión. Existe un pequeño porcentaje de pacientes que no responden al tratamiento convencional. Se denomina PTI refractaria a la trombocitopenia severa que persiste luego de realizada la esplenectomía, mientras que los pacientes que cumplen criterios de PTI severa pero que no han sido esplenectomizados se consideran "no respondedores" a los fármacos utilizados hasta ese momento. Se presentan tres casos clínicos de PTI severa, persistente y crónica, no respondedores al tratamiento, con el objetivo de señalar la importancia de una concienzuda pesquisa diagnóstica y el desarrollo de una eficaz combinación de tratamientos para el manejo de esta compleja patología.

Palabras clave: trombocitopenia inmune, refractaria, esplenectomía, hemorragia.

\begin{abstract}
Primary immune thrombocytopenia (ITP) is a rare pathology characterized by isolated thrombocytopenia of autoimmune origin, with a platelet count $<100 \times 109$ / L not associated with a recognizable pathology, with an increased risk of bleeding. There is no definitive confirmatory test to establish its diagnosis, which is carried out mainly by exclusion. There is a small percentage of patients who do not respond to conventional treatment. Refractory ITP refers to severe thrombocytopenia that persists after splenectomy, while patients who meet criteria for severe ITP but who have not undergone splenectomy are considered "non-responders" to the drugs used up to that time. Three clinical cases of severe, persistent and chronic ITP, non-responders to treatment, are presented with the aim of pointing out the importance of a thorough diagnostic investigation and the development of an effective combination of treatments for the management of this complex pathology.
\end{abstract}

Keywords: immune thrombocytopenia, refractory, splenectomy, hemorrhage.

Revista Methodo: Investigación Aplicada a las Ciencias Biológicas. Universidad Católica de Córdoba. Jacinto Ríos 571 Bo Gral. Paz. X5004FXS. Córdoba. Argentina. Tel.: (54) 3514517299 / Correo: methodo@ucc.edu.ar / Web: methodo.ucc.edu.ar | CASO CLINICO Rev. Methodo 2022;7(1):37-41. 


\section{Introducción}

La trombocitopenia inmune primaria (PTI) se caracteriza por trombocitopenia aislada de origen autoinmune, con un recuento $<100 \times 10^{9}$ plaquetas $/ 1$ no asociado a una patología reconocible ${ }^{1}$. Cursa con un aumento del riesgo de hemorragia, aunque no siempre hay manifestaciones hemorrágicas ${ }^{2}$. El mecanismo fisiopatológico principal es una desregulación de los linfocitos T que da lugar a una respuesta tanto celular como humoral contra antígenos plaquetarios y megacariocíticos ${ }^{3,4}$.

De acuerdo a criterios basados en temporalidad puede clasificarse como: de reciente diagnóstico (menos de tres meses a partir del diagnóstico), persistente (3-12 meses) y crónica (más de 12 meses $)^{2}$. La incidencia oscila entre 3,3 a 10 casos/100000 personas/año en adultos ${ }^{3}$. No existe ninguna prueba confirmatoria definitiva para establecer su diagnóstico, que se realiza fundamentalmente por exclusión, siendo la respuesta al tratamiento un parámetro de confirmación ${ }^{1}$.

No obstante, el tratamiento actual de PTI no está estrictamente definido. El criterio actual de respuesta exige obtener una cifra de plaquetas mayor o igual a $30 \times 10^{9} / 1$ con un incremento de más de dos veces la cifra basal y ausencia de hemorragia $^{1,2}$. La respuesta completa se define por un recuento mayor a $100 \times 10^{9} / 1$ y ausencia de hemorragia $^{1,2}$. Existe, sin embargo, un pequeño porcentaje de pacientes que no responden al tratamiento convencional. Se denomina PTI refractaria a la trombocitopenia severa que persiste luego de realizada la esplenectomía, mientras que los pacientes que cumplen criterios de PTI severa pero que no han sido esplenectomizados se consideran "no respondedores" a los fármacos utilizados hasta ese momento ${ }^{1}$. Se presentan a continuación tres casos clínicos de PTI severa no respondedores al tratamiento con las medidas realizadas en nuestra Institución, con el objetivo de señalar la importancia de una concienzuda pesquisa diagnóstica y el desarrollo de una combinación eficaz y oportuna de tratamientos, así como un estricto seguimiento clínico, para el manejo de estas entidades raras y sumamente complejas, todo lo cual implica un importante impacto en la calidad de vida de los pacientes.

\section{Caso 1}

Varón de 46 años, con antecedente de hipertensión arterial y obesidad mórbida, consulta por petequias múltiples localizadas en lengua y ambos miembros inferiores, de 24 horas de evolución, de aparición espontánea, sin otra sintomatología asociada. El paciente presentaba presión arterial de 140/100 $\mathrm{mmHg}$, petequias en miembros inferiores y en conjuntivas palpebrales; púrpura húmeda en cara ventral de la lengua; sin adenopatías ni visceromegalias. El laboratorio mostró: hemoglobina $=15,3 \mathrm{~g} / \mathrm{dl}$, hematocrito $=47,8 \%$, plaquetas $=1 \times 10^{9} / 1$, leucocitos $=8,1 \times 10^{9} / 1$, prueba de Coombs negativa. Las serologías reumatológicas y virales para $\mathrm{VIH}$, hepatitis B y C, Epstein Barr y Citomegalovirus fueron negativas. Las hormonas tiroideas y el proteinograma por electroforesis fueron normales. El antígeno para Helicobacter Pylori en materia fecal fue negativo. Inicia tratamiento con dexametasona $40 \mathrm{mg} /$ día endovenoso por cuatro días. Debido a una falta de respuesta terapéutica (plaquetas $1 \times 10^{9} / 1$ ) se realiza infusión de gammaglobulinas $(2 \mathrm{gr} / \mathrm{kg})$ durante dos días. Evoluciona con flictenas hemorrágicas en mucosa yugal, equimosis en conjuntiva tarsal y hematomas en ambos miembros superiores. La biopsia de médula ósea evidenció hipercelularidad, con conservación de la morfología y distribución de las tres series, sin cambios atípicos ni infiltración tumoral. La citometría de flujo no detectó población hematopoyética con perfil fenotípico anormal. Una tomografía de tórax, abdomen y pelvis constató hepatomegalia $13 \mathrm{~cm}$, bazo normal. Debido a la severidad del cuadro, inicia terapia con eltrombopag, con aumento progresivo de dosis hasta dosis máxima (75 mg/día) a los nueve días de iniciado dicho tratamiento. Evoluciona con cefalea, púrpura húmeda y episodios reiterados de epistaxis, con trombocitopenia severa persistente (plaquetas $1 \times 10^{9} / 1$ ), se indica adicionar al tratamiento instituido un segundo ciclo de dexametasona por cuatro días (a 14 días del primero). Posteriormente, por aumento de extensión de púrpura húmeda se indica meprednisona para disminuir el riesgo de sangrado espontáneo, a siete días de finalizado el segundo ciclo de corticoides. Habiendo completado 21 días de tratamiento con eltrombopag, persiste con trombocitopenia severa (plaquetas $5 \times 10^{9} / 1$ ), se rota a romiplostim (3 ug/kg), con mejoría del cuadro clínico y del valor de plaquetas $\left(108 \times 10^{9} / 1\right)$. Alta tras 36 días de internación, sin requerimiento posterior de medicación.

\section{Caso 2}

Varón de 45 años, sin antecedentes clínicos relevantes, consulta por petequias localizadas en abdomen y miembros inferiores, asociado a hematomas de tres días de evolución sin relación a 
traumatismo previo u otra sintomatología relevante. Presentaba petequias localizadas en paladar blando, los cuatro miembros, tórax $\mathrm{y}$ abdomen; hematomas en flancos, en miembro superior izquierdo y miembro inferior derecho. No se objetivaron adenopatías ni pérdida ponderal. El laboratorio mostró: hemoglobina $=14 \mathrm{~g} / \mathrm{dl}$, hematocrito $=40,4 \%, \quad$ leucocitos $=8,8 \times 10^{9} / 1$, plaquetas $=13 \times 10^{9} / 1, \mathrm{LDH}=512$. La ecografía de abdomen demostró esplenomegalia de $138 \mathrm{~mm}$. Las serologías reumatológicas y virales para VIH, hepatitis B y C, Epstein Barr, Citomegalovirus y Dengue fueron negativas. Las hormonas tiroideas y el proteinograma por electroforesis fueron normales. Inicia dexametasona $40 \mathrm{mg} /$ día por cuatro días y transfusión de nueve unidades de plaquetas por sangrado activo con mejoría del estado clínico y resolución de trombocitopenia severa (plaquetas $55 \times 10^{9} / 1$ ), con alta posterior. Al control a las 48 horas del alta hospitalaria, se constata recaída de trombocitopenia severa (plaquetas $1 \times 10^{9} / 1$ ), no respondedora a corticoterapia. Se indica infusión de gammaglobulinas (2 $\mathrm{gr} / \mathrm{kg})$ por dos días. El antígeno para Helicobacter Pylori en materia fecal fue negativo. La biopsia de médula ósea mostró hipercelularidad con megacariocitos presentes, sin cambios atípicos ni infiltración tumoral. Evoluciona con episodios de gingivorragia y púrpura húmeda, con persistencia de trombocitopenia severa (plaquetas $8 \times 10^{9} / 1$ ), se indica rescate con meprednisona, con respuesta parcial. Inicia terapia con eltrombopag ( $50 \mathrm{mg} /$ día) a 15 días del diagnóstico. Alta por mejoría del cuadro clínico, con trombocitopenia moderada (plaquetas $43 \times 10^{9} / 1$ ) con descenso progresivo de corticoides. En controles sucesivos hay una progresiva disminución en el conteo de plaquetas, se aumenta dosis de eltrombopag y meprednisona, sin obtenerse una respuesta significativa. Reingresa dos meses más tarde por trombocitopenia severa (plaquetas $12 \times 10^{9} / 1$ ) $\mathrm{sin}$ signos de sangrado activo, se rota a romiplostim (1 $\mathrm{ug} / \mathrm{kg}$ ), manteniendo meprednisona a altas dosis, con buena respuesta clínica y analítica (plaquetas $\left.46 \times 10^{9} / 1\right)$. Posterior recaída de trombocitopenia severa a las diez semanas (plaquetas $1 \times 10^{9} / 1$ ) con púrpura húmeda: inicia meprednisona $80 \mathrm{mg} /$ día y se aumenta dosis de romiplostim $(7 \mathrm{ug} / \mathrm{kg})$, con resolución del cuadro clínico y la trombocitopenia (plaquetas $308 \times 10^{9} / 1$ ).

\section{Caso 3}

Varón de 23 años, antecedente de PTI diagnosticada dos años previos al ingreso, con buena respuesta inicial a corticoides, consulta por petequias de 24 horas de evolución localizadas en miembros inferiores, sin sangrado activo espontáneo. Presentaba buen estado general; petequias en miembros inferiores y en mucosa yugal; hematomas en región de ambos muslos, hombro derecho; equimosis en abdomen. El hemograma mostró: hemoglobina $=14,2 \mathrm{~g} / \mathrm{dl}$, hematocrito $=42,5 \%$, leucocitos $=11,1 \times 10^{9} / 1$, plaquetas $=7 \times 10^{9} / 1$. Las serologías reumatológicas y virales para VIH y hepatitis B y C fueron negativas. El antígeno para Helicobacter Pylori en materia fecal fue negativo. Inicia dexametasona 40 $\mathrm{mg}$ /día por cuatro días. Debido a trombocitopenia severa persistente (plaquetas $9 \times 10^{9} / 1$ ), inicia meprednisona $60 \mathrm{mg} /$ día con posterior infusión de gammaglobulinas (2 $\mathrm{gr} / \mathrm{kg})$ en dos días. Evoluciona con mejoría de cuadro clínico y respuesta parcial inmediata a la terapia (plaquetas $\left.90 \times 10^{9} / 1\right)$, se otorga alta a los 10 días de internación. En controles sucesivos se observa trombocitopenia progresiva refractaria a terapias de primera línea, con indicación de esplenectomía, a la cual se niega. Reingresa a las seis semanas por trombocitopenia severa (plaquetas $19 \times 10^{9} / 1$ ). Al examen físico presentaba petequias en mucosa yugal, sin fiebre, adenopatías u otras alteraciones cutáneas. Hemograma: leucocitos $=12,1 \times 109 / 1$, eosinófilos $=1,69 \times 10^{9} / \mathrm{l}$. El dosaje de $\operatorname{IgE}$ fue normal. El coproparasitológico fue negativo. La biopsia de médula ósea evidenció hiperplasia de megacariocitos, eosinofilia medular, sin cambios atípicos ni infiltración tumoral. El proteinograma por electroforesis fue normal. Inicia rescate con gammaglobulinas por dos días con mejoría de plaquetas $\left(56 \times 10^{9} / 1\right)$. Se recibe eltrombopag e inicia tratamiento a dosis $50 \mathrm{mg} /$ día. Alta a los cuatro días de internación. En controles sucesivos, se constata disminución progresiva de plaquetas, con resolución posterior de eosinofilia: se aumenta dosis de eltrombopag (75mg/día) a cinco semanas de iniciado el tratamiento y se inicia meprednisona $80 \mathrm{mg} /$ día. Evolución favorable, se disminuye progresivamente dosis de eltrombopag hasta 25 mg diarios.

\section{Discusión}

La trombocitopenia inmune primaria (PTI) se caracteriza por trombocitopenia aislada de origen autoinmune $^{1}$. Afecta a todas las edades, con una incidencia anual de 3,3/100000 adultos que aumenta con la edad ${ }^{3}$, con predominio en mujeres en edad reproductiva, si bien los tres casos clínicos corresponden a adultos jóvenes de sexo masculino $^{3,5}$.

No existe ninguna prueba confirmatoria para establecer su diagnóstico, que se realiza 
fundamentalmente por exclusión, por lo que deben descartarse causas no inmunes y hereditarias, así como las PTI secundarias ${ }^{3,4,6}$. En el caso de nuestros tres pacientes, se realizó punción aspirativa de médula ósea al no obtenerse una respuesta al tratamiento inicial ${ }^{1}$, si bien este método se encuentra indicado durante la evaluación inicial si la plaquetopenia se acompaña de otras citopenias o en el caso de adultos mayores a 60 años, así como previo a indicar una esplenectomía ${ }^{1}$.

El tratamiento actual de PTI no está estrictamente definido. En nuestros tres casos se inició

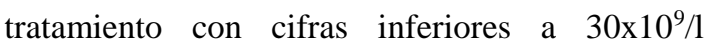
plaquetas ${ }^{1}$. Casos con 30-50x $10^{9} / 1$ plaquetas deben tratarse ante sangrado o en caso de procedimiento, como así también en aquellos casos seleccionados con un estilo de vida de riesgo ${ }^{1}$. El tratamiento de primera línea suele consistir en corticoesteroides (dexametasona o prednisona en dosis altas) ${ }^{7}$ o inmunoglobulina endovenosa ${ }^{8,9}$. Si se observa una respuesta positiva, está recomendado iniciar un descenso gradual de corticoides con el objetivo de suspenderlo a las seis semanas (máximo ocho semanas $)^{8}$. Si no hay respuesta a la dosis inicial dentro de las dos semanas, se sugiere disminuir gradualmente el corticoide durante una semana para finalmente discontinuar el tratamiento ${ }^{8}$. Por otro lado, las inmunoglobulinas se prefieren antes que la corticoterapia en pacientes con sangrado activo, alto riesgo de sangrado o aquellos con requerimiento de procedimientos quirúrgicos ${ }^{8}$.

El tratamiento de segunda línea incluye fundamentalmente la esplenectomía, rituximab y los agonistas del receptor de la trombopoyetina (AR-TPO): eltrombopag y romiplostim ${ }^{1,2,3,8,9}$. No existen pautas para especificar el orden en el que se deben utilizar dichos agentes6. Actualmente, tanto eltrombopag como romiplostim se encuentran aprobados para el tratamiento de pacientes no respondedores ${ }^{10,11}$, mientras que rituximab constituye una indicación no aprobada para PTI, si bien existen numerosas publicaciones que avalan su utilidad, aunque ningún estudio aleatorizado ${ }^{10}$. Por lo tanto, el tratamiento debe individualizarse en función de la duración de la PTI, la frecuencia de episodios hemorrágicos, comorbilidades, adherencia a la medicación, preferencias del paciente, costo y disponibilidad ${ }^{2}$. A su vez, tal como se describe en el tercer caso, una recaída tardía autoriza a repetir un esquema similar al inicial ${ }^{1}$.

La esplenectomía es hoy el tratamiento más eficaz a largo plazo, el cual se difiere hasta un año después de realizado el diagnóstico, tal como se señala en el tercer caso, dada la posibilidad de remisiones espontáneas y estabilización plaquetaria $^{1}$. Hasta un tercio de los pacientes pueden remitir en un año y hasta el $80 \%$ pueden remitir en cinco años. Los riesgos a largo plazo incluyen aumento de las tasas de trombosis, infecciones y cáncer ${ }^{8}$, mientras que a su vez la morbilidad aumenta en relación con factores como la obesidad, la edad avanzada y la pluripatología crónica ${ }^{2}$.

En nuestra serie de casos, siendo los dos primeros pacientes correspondientes a enfermedad persistente, tras la falta de respuesta a las terapias de primera línea, se emplearon preferentemente los AR-TPO como primer agente de segunda línea. Está descrita una mayor durabilidad en la respuesta con el uso continuado de estos agentes respecto a rituximab $^{8}$. Por otro lado, pacientes con PTI crónica en los que se prioriza una respuesta duradera, las opciones principales recomendadas son la esplenectomía y los AR-TPO ${ }^{8}$.

Se ha determinado que no existe una diferencia significativa respecto a los beneficios netos o el perfil de seguridad entre la administración de eltrombopag y romiplostim ${ }^{8,10}$, por lo que la toma de decisiones iniciales podría verse impulsada por la preferencia del paciente por la vía de administración, es decir, la medicación diaria oral (eltrombopag, en dosis de $25-75 \mathrm{mg} /$ día) en comparación con la inyección subcutánea semanal (romiplostim, en dosis de 1-10 ug/kg), así como por las características referentes al costo $\mathrm{y}$ disponibilidad de cada uno en particular ${ }^{8}$.

Para concluir, siendo que los tres casos corresponden a PTI no respondedora con parámetros de trombocitopenia severa con un alto riesgo de sangrado, resulta fundamental alternar terapias combinadas de acuerdo a su evolución (corticoterapia y AR-TPO), con diferentes mecanismos de acción y toxicidades primarias, con el objetivo de optimizar la efectividad del tratamiento ${ }^{6}$.

\section{Bibliografía}

1. Fantl D, Milovic V, Shanley C, et al. Guías de diagnóstico y tratamiento: edición 2019. [Internet] 1a ed revisada. - Ciudad Autónoma de Buenos Aires (Arg): Sociedad Argentina de Hematología; 2019. Disponible en: www.sah.org.ar/docs/2019/Guia_2019completa.pdf; consultado octubre 2020.

2. Sanz Alonso MA, García VV. Directrices de diagnóstico, tratamiento y seguimiento de la PTI: Documento de Consenso. [Internet]. 1a ed. Madrid: Sociedad Española de Hematología y Hemoterapia; 2011. Disponible en: www.sehh.es/publicaciones/consensos/174 
3-directrices-de-diagnostico-tratamiento-yseguimiento-de-la-pti-documento-deconsenso; consultado octubre 2020.

3. Beligoy LE. Trombocitopenia inmune persistente y crónica del adulto: opciones terapéuticas. Hematología. 2018; 22 (Extraord): 54-61.

4. Wilson RG. Diagnóstico y tratamiento de la púrpura trombocitopénica inmunológica. Rev Med Hered. 2015; 26 (4):246-255.

5. Moulis G, Lapeyre-Mestre M, Adoue D, Sailler L. Épidémiologie et pharmacoépidémiologie du purpura thrombopénique immunologique [Epidemiology and pharmacoepidemiology of immune thrombocytopenia]. Rev Med Interne. $2017 \mathrm{Jul} ; 38$ (7):444-449.

6. Miltiadous O, Hou M, Bussel JB. Identifying and treating refractory ITP: difficulty in diagnosis and role of combination treatment. Blood. 2020; 135(7): 472-490.
7. Samson M, Fraser W, Lebowitz D. Treatments for Primary Immune Thrombocytopenia: A Review. Cureus. 2019; 11(10): e5849.

8. Neunert C, Terrell DR, Arnold DM, et al. American Society of Hematology 2019 guidelines for immune thrombocytopenia. Blood Adv. 2019; 3 (23): 3829-3866.

9. Witkowski M, Witkowska M, Robak T. Autoimmune thrombocytopenia: Current treatment options in adults with a focus on novel drugs. Eur J Haematol. 2019;103 (6):531-541.

10. Fondevila CG. Tratamiento de segunda línea de la Trombocitopenia Inmune del adulto. Hematología. 2014; 18 (2): 133-139.

11. Petro K, et al. Púrpura trombocitopénica inmune: tratamiento de segunda línea, experiencia en el Sanatorio Güemes centrada en trombomiméticos. Hematología. 2020; 24(3): 19-24.

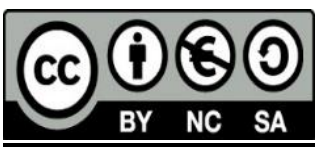

\section{Anexo tabla}

Tabla 1. Características de los pacientes

\begin{tabular}{|c|c|c|c|}
\hline & CASO 1 & CASO 2 & CASO 3 \\
\hline Sexo & Masculino & Masculino & Masculino \\
\hline Edad & 46 & 45 & No \\
\hline Comorbilidades & Hipertensión arterial - Obesidad mórbida & No & No \\
\hline Sangrado activo & Durante la evolución & Al inicio y durante la evolución & Hígado 18,2 cm \\
Bazo 13,3 cm
\end{tabular}

